\title{
ON THE SPREADING AND SOLIDIFICATION OF MOLTEN PARTICLES IN A PLASMA SPRAY PROCESS : EFFECT OF THERMAL CONTACT RESISTANCE
}

\author{
M. Pasandideh-Fard and J. Mostaghimi \\ University of Toronto \\ Department of Mechanical Engineering \\ 5 King's College Road, Toronto, Ontario, Canada M5S IA4
}

The spreading and simultaneous solidification of a liquid droplet upon its impingement onto a substrate permitting thermal contact resistance has been numerically simulated; the effect of contact resistance and the importance of solidification on droplet spreading are investigated. The numerical solution for the complete Navier-Stokes equations is based on the modified SOLA-VOF method using rectangular mesh in axisymmetric geometry. The solidification of the deforming droplet is considered by a one-dimensional heat conduction model. The predictions are in good agreement with the available experimental data and the model may be well suited for investigating droplet impact and simultaneous solidification permitting contact resistance at the substrate. We found that the final splat diameter could be extremely sensitive to the magnitude of the thermal contact resistance. The results also show that for the condition of higher Reynolds and/or higher Stefan numbers the effect of solidification on the final splat diameter is more important.

\section{INTRODUCTION}

Fluid flow and heat transfer phenomena associated with the impingement, spreading, and solidification of liquid droplets are of broad importance in a number of materials processing applications. Typical examples include thermal plasma spraying of ceramics and metallic materials, high velocity oxy-fuel (HVOF) spraying, and spray forming processes such as Osprey spray forming process.

This is a complicated problem because it involves substantial deformation of the droplet and simultaneous solidification within a short time. In this paper we focus on the prediction of deformation and 
solidification of liquid droplets under conditions typical of thermal plasma spraying processes. In a plasma spray process, material powders are injected into a high temperature plasma where they are rapidly melted and accelerated before being deposited onto a substrate. The mechanical properties of plasma-sprayed coatings depend to a large extent on the details of the spraying process, in particular, they are strongly dependent on the solidification and deformation history of the individual droplets.

Simple analytical models of droplet impact have been proposed based on an energy balance that equates initial droplet kinetic energy to the change in surface energy due to droplet deformation and the work done in overcoming viscosity during impact. Several such models have been reviewed by Bennett and Poulikakos ${ }^{(1)}$. However, calculations of heat transfer between the surface and droplet require detailed information about droplet shape during impact, which can be obtained only by a complete numerical solution of the continuity, momentum and energy equations. To this end, several numerical simulations for the isothermal droplet impaction process can be found in the literature. These models and their applicability have been discussed elsewhere ${ }^{(2)}$. Bennett $\&$ Poulikakos ${ }^{(3)}$ and Kang et al ${ }^{(4)}$ studied the solidification behavior of droplet impact based on an assumption that solidification starts when spreading is completed. The droplet first deforms to its maximum spread in the form of a flat disc, then a one dimensional energy equation for the disc is solved. The validity of such an assumption depends on the impact of solidification on droplet spreading which is one contribution of the present paper. In a simplified approach, Madejski ${ }^{(5)}$ analytically investigated the deformation and solidification of a single molten droplet in a plasma spray process. His analytical formulae provide an estimate of the final degree of spreading, but provide no information regarding the dynamics of the deformation process. Marchi et al. ${ }^{(6)}$ considered the numerical solution of Madejski's theoretical analysis. However, the importance of solidification is not discussed. In addition, the theoretical solution assumes no thermal contact resistance at the substrate. Watanabe et al. ${ }^{(7)}$, Liu et al. ${ }^{(89.10)}$ and Trapaga et al. ${ }^{(11)}$ employed a computational method to study the simultaneous solidification. No thermal contact resistance has been incorporated in their model. Moreover, the effect of solidification on droplet spreading is not addressed.

The splat formation and cooling rate of plasma sprayed molybdenum particles on different substrates were investigated experimentally by Moreau et al. ${ }^{(12,13,14)}$. Their work is concerned with the influence of substrate conditions and materials on the coatings texture and the cooling rate of sprayed particles. There is a large scatter in their measurements which is the result of a very difficult experimental conditions. Their measurements indicate that surface conditions play an important role in the solidification process. Fantassi et al. ${ }^{(15)}$, Bianchi et al. ${ }^{(16.17}$ and Vardelle et al. ${ }^{(18)}$ using a different experimental set up, came to similar conclusions.

In the present study, the transient behaviour of deformation and simultaneous solidification of a single droplet upon impingement onto a 
substrate permitting thermal contact resistance is numerically simulated; the effect of contact resistance and the importance of solidification on the spreading are addressed. A comparison between numerical results and the available experimental data is performed to validate the model.

\section{MATHEMATICAL FORMULATIONS}

A schematic diagram of a liquid droplet at the time of impact, $t=0$, is shown in Fig.l. It is reasonable to assume that during the spreading, the temperature gradient is much larger in the axial direction and thus, the heat transfer problem can be approximated by a one-dimensional model. The mathematical model developed in this paper is based on the assumptions of : laminar and incompressible fluid flow, axisymmetric system of coordinates, vertical impingement on the substrate, one-dimensional energy equation for solidification, and negligible convection and radiation heat transfer ${ }^{(19,20)}$. We also assume that the surface of the substrate is smooth, homogeneous, isotropic and insoluble. It should be noted that in plasma spraying the substrate is usually rough and oxidized. This represents an additional resistance to heat transfer between droplet and substrate. Based on the above assumptions, the governing flow equations are the classical Navier-Stokes equations

$$
\begin{gathered}
\nabla \cdot \boldsymbol{V}=0 \\
\frac{\partial \boldsymbol{V}}{\partial t}+(\boldsymbol{V} \cdot \nabla) \boldsymbol{V}=\frac{-1}{\rho} \nabla P+v \nabla^{2} \boldsymbol{V}+\boldsymbol{g}
\end{gathered}
$$

where $V$ is velocity vector; $P, \rho$ and $v$ are pressure, density and

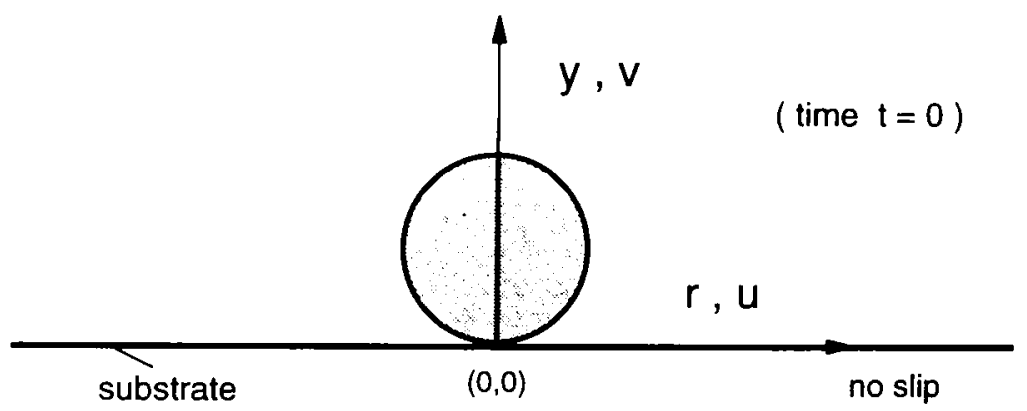

Fig.1. Initial configuration used in numerical computations. 
kinematic viscosity of the fluid, respectively, and $g$ represents the body force per unit mass.

To predict the surface profile of the deforming droplet, we use the "fractional volume of fluid" scheme. In this technique, a scalar function $F$, is defined whose value is equal to the fractional volume of the cell occupied by the fluid ${ }^{(21)} . F$ is assumed to be unity when a cell is fully occupied by the fluid and zero for an empty cell. Cells with values of $0<F<1$ contain a free surface. With this definition, function $F$ is conserved through the following equation

$$
\frac{\partial F}{\partial t}+(\boldsymbol{V} \cdot \nabla) F=0
$$

Energy equation(solidification):

We assume the substrate to have a high thermal conductivity, e.g. metallic substrates, therefore, the substrate may be considered as a reservoir with a constant temperature. Denoting the liquid phase with an index of $L$, the solid phase with $S$ and assuming that the solidification problem can be approximated by a one dimensional model similar to Stefan problem ${ }^{(22)}$, the equations of heat conduction can be written as

$$
\begin{array}{ll}
\frac{\partial T_{L}}{\partial t}=\alpha_{L} \cdot \frac{\partial^{2} T_{L}}{\partial y^{2}} & , \quad y>\delta(t) \\
\frac{\partial T_{S}}{\partial t}=\alpha_{S} \cdot \frac{\partial^{2} T_{S}}{\partial y^{2}} & , \quad 0 \leq y \leq \delta(t)
\end{array}
$$

where $T$ and $\alpha$ are the temperature and thermal diffusivity, respectively, and $\delta(t)$ represents the thickness of the solidified layer at time $t$.

Boundary conditions for the flow problem are shown in Fig.1. The flow is assumed to be axisymmetric with no slip at the solid substrate. At a free surface, tangential stresses are assumed to be negligible. Normal stresses at a free surface are replaced by an equivalent surface pressure, calculated from the interface mechanical equilibrium condition given by the Laplace equation of capillarity ${ }^{(23)}$

$$
P_{1}-P_{v}=J \gamma
$$

where $P_{l}$ and $P_{1}$ are pressures inside and outside the droplet, respectively, $J$ is the interface mean curvature and $\gamma$ represents the liquid-gas surface tension.

Describing the liquid-solid contact line requires special attention. The 
contact angle, $\theta$, is incorporated in the analysis to obtain the mean curvature $J$ of the menisci near the substrate. The model uses either a constant value of $\theta$, or a dynamic contact angle that varies with time during droplet impact.

The boundary and initial conditions for the energy equations are

$$
\begin{gathered}
y \rightarrow \infty ; T_{L}=T_{\infty}>T_{m p} \quad, \quad y=0 ; \quad T_{S}=T_{o}<T_{m p} \\
@ t=0: T_{L}=T_{\infty}
\end{gathered}
$$

where $T_{\infty}$ and $T_{0}$ are the temperature of droplet and substrate before impact, respectively, and $T_{m p}$ represents the melting point temperature. When thermal contact resistance at the substrate is considered, the boundary condition at $y=0$ is modified as follows

$$
\text { @ } y=0 \quad: \quad T_{S}=T_{,}+K_{S} R_{t, c} \frac{\partial T_{S}}{\partial y}
$$

where $K_{S}$ is the solid thermal conductivity and $R_{t, c}^{*}$ represents the thermal contact resistance at the substrate per unit area. There are two other boundary conditions for the temperature equality and the conduction heat balance at the solid-liquid interface.

\section{COMPUTATIONAL PROCEDURE}

The governing equations are solved numerically using a modified SOLA-VOF algorithm. The algorithm is based on the "Marker-and-Cell" finite-difference technique. Most terms in the momentum equations are evaluated using an explicit computational scheme. The coupling between the pressure and velocity is, however, assumed to be implicit. The resultant semi-implicit, finite difference equations are solved using the successive over-relaxation method.

For the solidification process, the following technique is employed. The analytical solution for the one-dimensional heat conduction problem described in the previous section is ${ }^{(22)}$ :

$$
\delta(t)=2 \Omega\left(\alpha_{s} t\right)^{1 / 2}
$$

where $\Omega$ is a constant obtained from the following equation :

$$
\frac{e^{-\Omega^{2}} S t e_{S}}{\operatorname{erf}(\Omega)}-\frac{\rho_{L}}{\rho_{S} b} \frac{e^{-\Omega^{2} b^{2}} S t e_{L}}{\operatorname{erfc}(\Omega b)}=\Omega \sqrt{\pi}
$$




$$
b=\left(\frac{\alpha_{S}}{\alpha_{L}}\right)^{1 / 2} \quad, \quad S t e_{S}=\frac{C_{S}\left(T_{m p}-T_{0}\right)}{\lambda} \quad \text { Ste } e_{L}=\frac{C_{L}\left(T_{\infty}-T_{m p}\right)}{\lambda}
$$

Ste is the Stefan number, and $C$ and $\lambda$ are the specific heat and heat of fusion, respectively. When thermal contact resistance is involved, $T_{S}$ is related to its own gradient with respect to $y$ at $y=0$, therefore, an iteration will be required to obtain $\Omega$ at every time $t$. To employ these results for the treatment of solidification in the simulation code, a computational procedure based on obtaining the location of the solid-liquid interface is followed. At each time increment $\delta t$, computation of the fluid flow problem is followed by calculation of the position of solidified layer. Based on the model, the solidification in a column of fluid starts when the control volume next to the substrate in that column is filled by the fluid. Having determined the starting time of the solidification in a column of fluid, the thickness of the solidified layer in the column is computed based on the associated time of solidification and the corresponding $\Omega$. The velocity and pressure associated with cells containing a solidified layer are set to zero, i.e. they no longer contribute to the process of flattening. Following the computation of the solidification front for the whole calculation domain, the boundary conditions for the fluid flow are readjusted and the time is advanced by a time increment. Computation of spreading and solidification is continued until the whole splat is solidified.

\section{RESULTS AND DISCUSSION}

First, we present the results for two cases representative of DC and RF plasma spraying conditions. Next, the results of the present model is compared with the available experimental measurements. The effect of contact resistance on droplet impact and the importance of solidification on the spreading will then be investigated.

\subsection{Results for Some Typical Spraying Conditions}

Results are presented for three different cases and two different materials, i.e., alumina and tin. Table 1 lists the initial droplet diameter $\left(D_{\mathrm{o}}\right)$, the impinging velocity $\left(V_{\mathrm{o}}\right)$, and the corresponding Reynolds $(R e)$, Weber (We) and Stefan (Ste) numbers for each case. Reynolds, Weber and Stefan numbers are defined as : $R e=D_{0} V_{0} v, W e=\rho D_{o} V_{0}^{2} / \gamma$ and Ste $=C_{S}\left(T_{m p}-T_{o}\right) / \lambda$. The initial velocities for cases 1 and 2 are typical of the impinging velocities of particles in atmospheric radio frequency inductively coupled plasma (RF-ICP) and DC thermal plasma spraying operations, respectively. We assume that the alumina droplets are at $500 \mathrm{~K}$ over the melting point temperature and the tin droplet is at $270 \mathrm{~K}$ over its melting 


\begin{tabular}{c|c|c|c|c|c|c} 
Case & Material & $\mathrm{D}_{\mathrm{o}}[\mu \mathrm{m}]$ & $\mathrm{V}_{\mathrm{o}}[\mathrm{m} / \mathrm{s}]$ & $\mathrm{Re}$ & $\mathrm{We}$ & Ste \\
\hline \hline 1 & alumina & 50 & 50 & 245 & 705 & 2.16 \\
\hline 2 & alumina & 50 & 200 & 975 & 11305 & 2.16 \\
\hline 3 & tin & 2700 & 3.704 & 35339 & 447 & 0.85
\end{tabular}

Table 1. Input parameters for droplet spreading and solidification in thermal spray operations

point temperature at the time of impact. The temperature of the substrate is considered to be $500 \mathrm{~K}$ for cases 1 and 2 and $293 \mathrm{~K}$ for case 3. No thermal contact resistance has been used for the first two cases. In addition, for all cases, we assume a constant contact angle of $\theta=90$ degrees, to represent the wetting behaviour. It has been shown elsewhere ${ }^{(2)}$ that under the conditions typical of thermal spray process, the assumption of constant contact angle is justified.

Computer-generated images of the spreading of an alumina droplet (Case 1, RF plasma spraying) at a viewing angle of $30^{\circ}$ with respect to the horizontal surface are shown in Fig.2. The liquid phase is indicated in gray while the solidified layer is indicated in black. In the early stages of deformation, the axial velocity of the fluid near the substrate becomes zero while the radial velocity is increased rapidly. A smooth convex shape which is due to the high viscosity of alumina is formed at the splat edge. In the early stages of deformation, the solidified layer is restricted to a very thin layer next to the substrate, therefore, the flattening process is not considerably affected by the solidification process. As time increases, the effect of solidification becomes more important. The effect appears as a reduction in the rate of increase in the splat diameter. The spreading of the droplet is finally arrested after $30 \mu \mathrm{s}$ when the whole droplet is almost solidified. After nearly $4 \mu s$, the diameter of the solidified layer remains constant, i.e. the spreading is completed in a much shorter time. The final diameter of the solidified layer is $0.122 \mathrm{~mm}$ and its final thickness is around $0.005 \mathrm{~mm}(5 \mu \mathrm{m})$. The solidification time for an alumina droplet in a typical RF-ICP spraying operation is in the order of $10 \mu s$.

Fig. 3 shows the spreading of a $50 \mu \mathrm{m}$ alumina droplet with an initial velocity of $200 \mathrm{~m} / \mathrm{s}$ impinging on a flat surface (Case 2, DC plasma spraying). In comparison with Case 1, the spreading develops faster because of the higher impact velocity. However, the basic deformation 

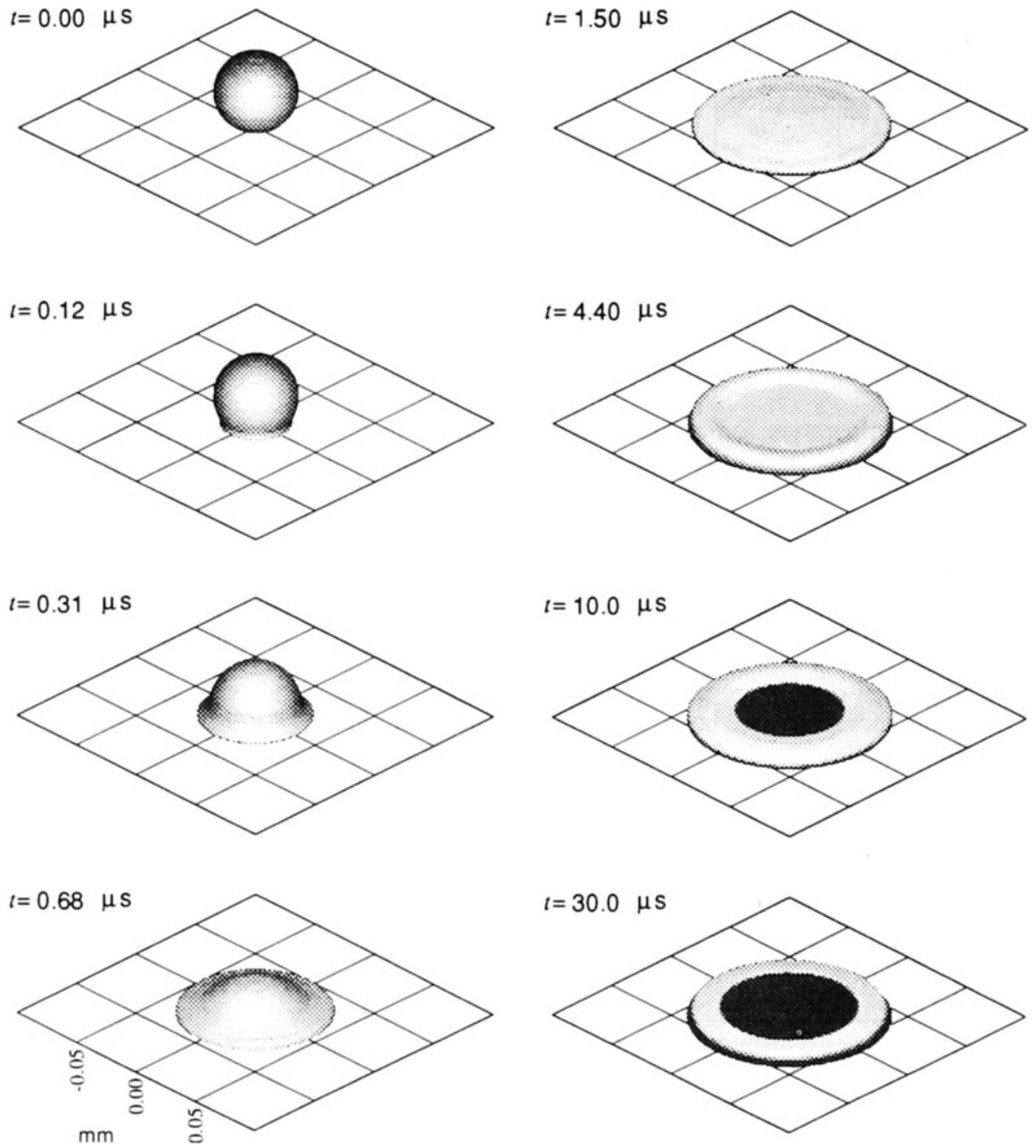

Fig.2. Sequence of the spreading and simultaneous solidification of an alumina droplet of Case 1 of Table I frepresenting a typical RF plasma spray operation). 

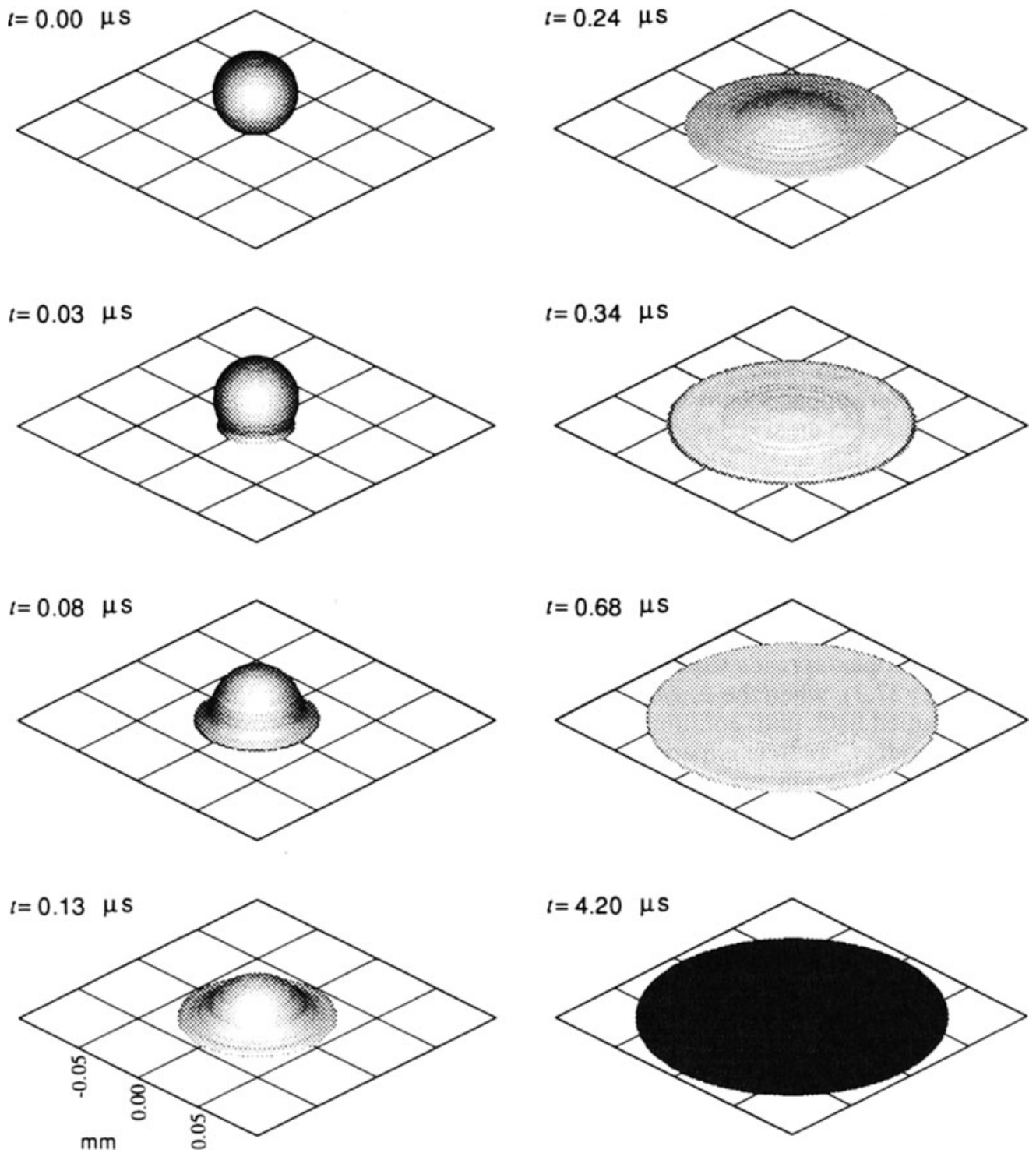

Fig.3. Sequence of the spreading and simultaneous solidification of an alumina droplet of Case 2 of Table 1 (representing a typical DC plasma spray operation). 
behaviour for the two cases is similar. After nearly $4 \mu s$, the process is completed and a solidified layer of $0.187 \mathrm{~mm}$ in diameter and 0.0025 $\mathrm{mm}(2.5 \mu \mathrm{m})$ in thickness is formed. Comparing Fig. 2 and Fig. 3 shows that the spreading process for Case 2 is approximately 4 times faster than Case 1 , a factor which is equal to their impact velocity ratio. We also note that the lamellae formed in Case 2 has a smooth surface which is a reflection of the high impact velocity.

\subsection{Comparison with Experimental Measurements}

We compare the results of numerical model with experiments performed by Chandra ${ }^{(2+)}$ under the condition of low We numbers for the isothermal spreading of a water droplet at room temperature upon its impingement onto a flat substrate. A single water droplet, $2.0 \mathrm{~mm}$ in diameter, impacts onto a stainless steel surface with a vertical velocity of $1 \mathrm{~m} / \mathrm{s}$. From enlarged photographs of droplet impact and spreading, liquidsolid contact angle and diameter of the wetted surface area are measured. The extent of droplet spreading is characterized by normalizing this diameter by the initial droplet diameter $\left(D_{c}\right)$ yields the so-called 'spreading factor', $\xi(t)=D(t) / D_{o}$. For the purpose of numerical simulation we use the equilibrium contact angle which is 90 degrees for this case as indicated in Ref.(24). Fig.4 compares the predicted and measured spreading factor vs. dimensionless time, $t^{*}=t V_{t} / D_{t}$, where $t$ is the elapsed time after impact. The droplet spreads to some extent but because of a very low We number ( $W e=27.3$ ), when the surface tension effects overcome the inertia, the fluid is pulled back until equilibrium condition is reached. Numerical simulation accurately predicts experimental measurements during droplet spreading. This agreement validates the assumptions made in formulating the numerical model. However, during droplet recoil there is considerable discrepancy between numerical predictions and measured values of $\xi$. The reasons for this are unclear, but it should be noted that as the droplet recedes it leaves a very thin liquid film behind on the surface. Modelling fluid flow realistically in this thin layer near the contact line presents considerable challenges. It is also possible that the apparent contact angle measured from photographs differs from the actual contact angle close to the solid surface ${ }^{(25)}$.

\subsection{Effect of Contact Resistance on Droplet Impact}

To study the effect of contact resistance on droplet impact, we consider Case 3 of Table 1, a case for which the experimental results are available in the literature. The transient deformation and simultaneous freezing of superheated tin droplets upon their impingement onto a flat substrate was studied experimentally by Fukanuma and Ohmori ${ }^{(26)}$. A single droplet, $2.7 \mathrm{~mm}$ in diameter and at $773 \mathrm{~K}$, impacts onto a flat, polished alumina substrate at $293 \mathrm{~K}$ with a vertical velocity of $3.704 \mathrm{~m} / \mathrm{s}$ 


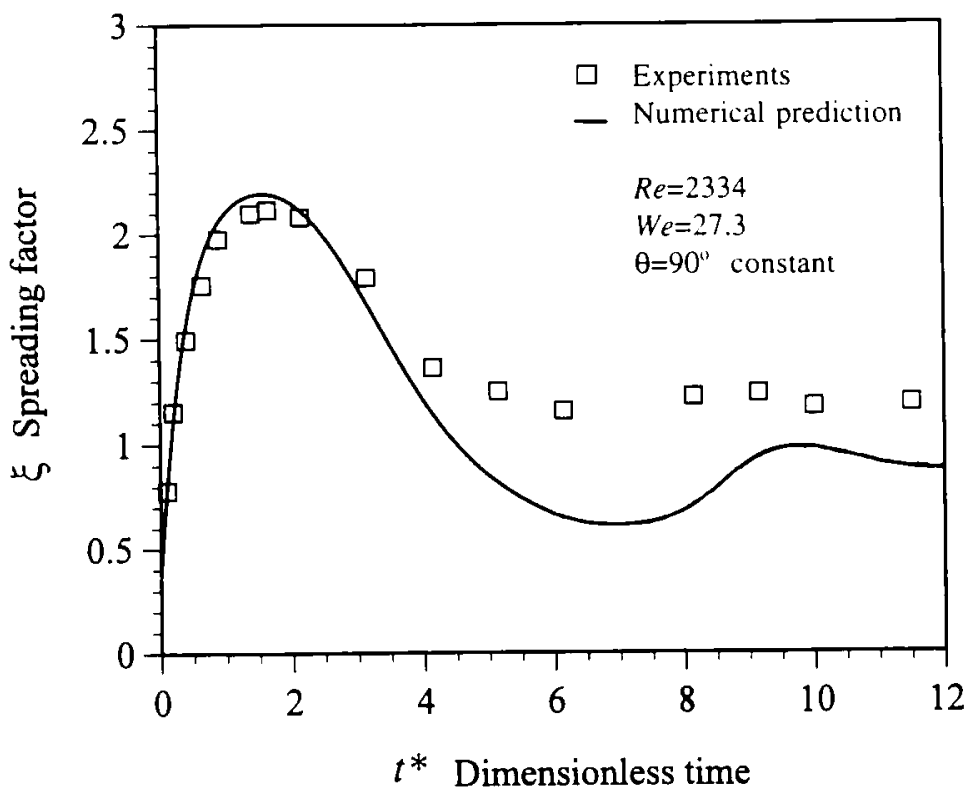

Fig.4. Comparison between experimental observations and simulation results for the isothermal spreading of a $2 \mathrm{~mm}$ water droplet impacting onto a substrate with the velocity of $1 \mathrm{~m} / \mathrm{s}$. Experimental results were adapted from Ref.(25).

(Case 3, Table 1). The disk diameters in the photographs (taken by a highspeed camera) were measured as the reference length of the solidified splat diameters. For the purpose of numerical simulation we consider different cases with different substrate conditions.

Fig.5 compares the predicted and measured spreading factor against time for the tin droplet under consideration. Simulation results are given for four different conditions at the substrate. When thermal contact resistance $\left(R_{t, c}^{-}\right)$is lower than $5 \times 10^{-7} \mathrm{~m}^{2} \mathrm{KW}^{-1}$ (curve (a) of Fig.5), solidification behavior is the same as when no contact resistance is considered. As $R_{t, c}^{-}$is increased, heat transfer to the substrate is reduced and, therefore, spreading factor is increased. For a contact resistance of $1.2 \times 10^{-6} \mathrm{~m}^{2} \mathrm{KW} W^{\Gamma}$, the simulation results agree with the experiments ${ }^{(26)}$. This, indicates the approximate value of thermal contact resistance for the case under consideration. Increasing contact resistance to $1.3 \times 10^{-6}$ $m^{2} K W^{l}$ produced a significant change in the shape of solidified splat. When $R_{t, c}$ is larger than $2 \times 10^{-6} \mathrm{~m}^{2} \mathrm{KW} \mathrm{W}^{-1}$, solidification does not change spreading factor compared to the isothermal case. Fig.6 shows the variation of the final spreading factor, $\xi_{\mathrm{m}}$, vs. the thermal contact resistance. For the particular case considered here (tin droplet on an 


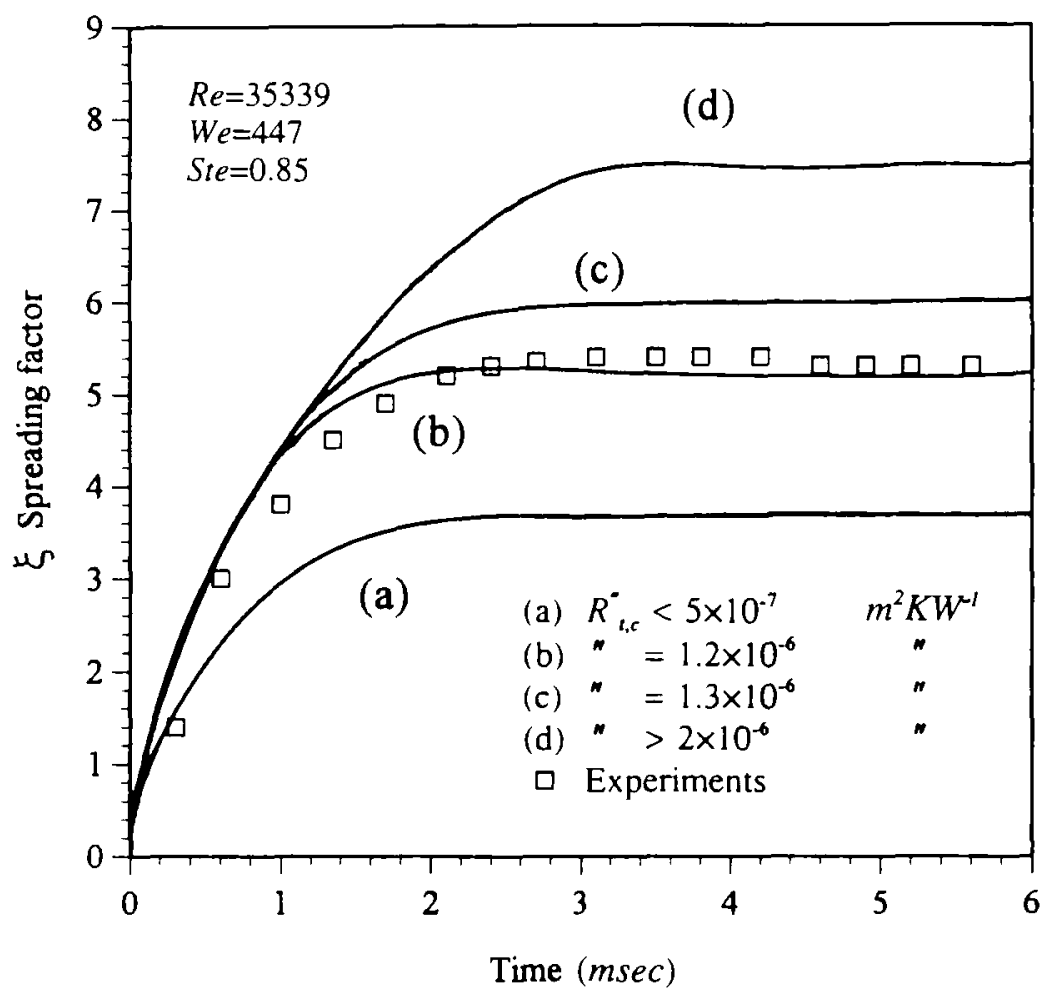

Fig.5. Comparison between experimental observations and simulation results for the spreading and simultaneous solidification of a tin droplet of Case 3 of Table 1. Computational results are given for different values of contact resistance at the substrate surface. Experimental results were adapted from Ref.(26).

alumina substrate), the final spreading factor, and thus the final splat diameter, is extremely sensitive to the magnitude of the thermal contact resistance (around $R_{b, c}^{-}=1 \times 10^{-6} \mathrm{~m}^{2} \mathrm{KW}^{-1}$ ). This finding is in accordance with the experimental measurements ${ }^{(12,27)}$ which have shown that the magnitude of the contact resistance for different droplet/substrate materials lies in a range between $5 \times 10^{-7}-5 \times 10^{-6} \mathrm{~m}^{2} \mathrm{KW}^{-1}$. The experiments have also confirmed the sensitivity of the final splat diameter to the substrate contact resistance.

\subsection{Effect of Solidification on Droplet Impact}

Most studies have neglected the possible effects of solidification on the spreading factor ${ }^{(1,3,4)}$. To study this effect, for the alumina droplet of Case 


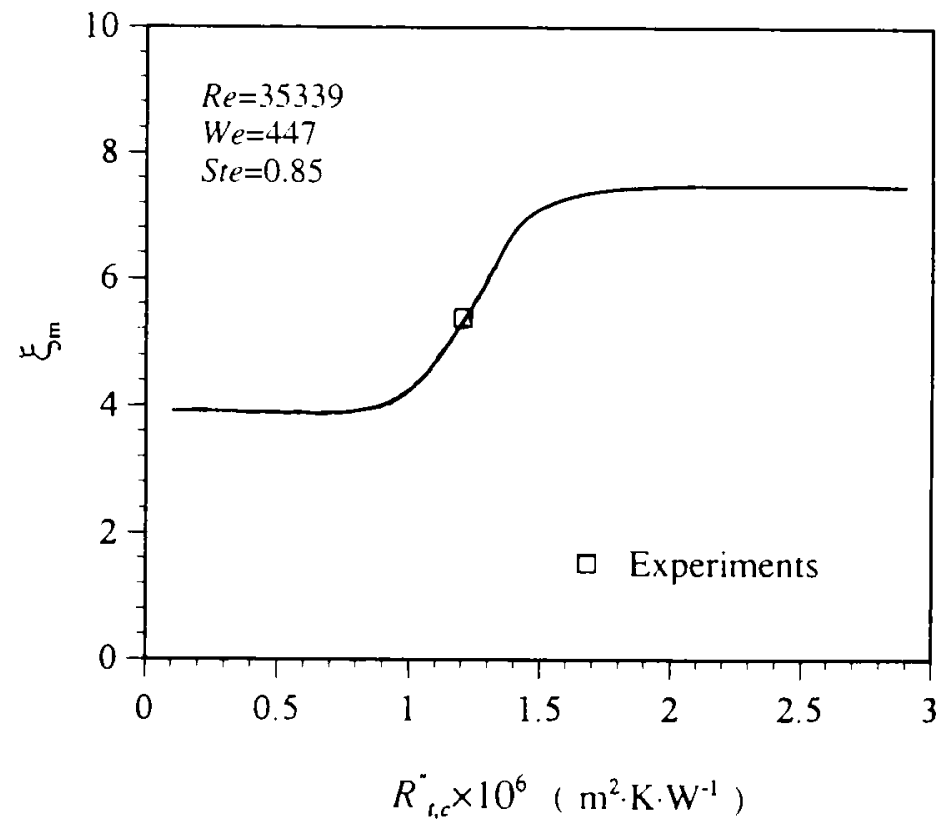

Fig.6. Variation of the final spreading factor, $\xi_{m}$, vs. the substrate thermal-contactresistance per unit area, $R_{h c}^{-}$, for the tin droplet under consideration on an alumina substrate. Experimental results were adapted from Ref.(26).

2 (Table 1), we considered : I) no solidification during the spreading and, II) simultaneous solidification and deformation. Fig.7a shows the variation of the spreading factor, $\xi$, for these considerations. The spreading factor is somewhat smaller when simultaneous solidification is considered. The decrease in $\xi$ is, however, not substantial (about 10\%) and the effect of solidification on the spreading behaviour is negligible for this particular case. Similar calculations were carried out for the tin droplet of Case 3 (Table 1). Fig.7b shows that in this case, the effect of solidification on arresting the spread of the droplet is quite substantial and neglecting this effect will result in a large error.

The relative importance of solidification on the spreading behaviour depends on both Reynolds and Stefan numbers. Higher $R e$ indicates faster spreading, while higher Ste reflects faster solidification. Thus, for conditions of high $R e$ and/or high Ste numbers, the solidification process plays a crucial role in arresting the spreading of the droplet, and its effect can not be neglected. A comparison between Case 2 and Case 3 shows that while Ste numbers are close, Re number for Case 2 is almost two orders of magnitude smaller than Case 3. 


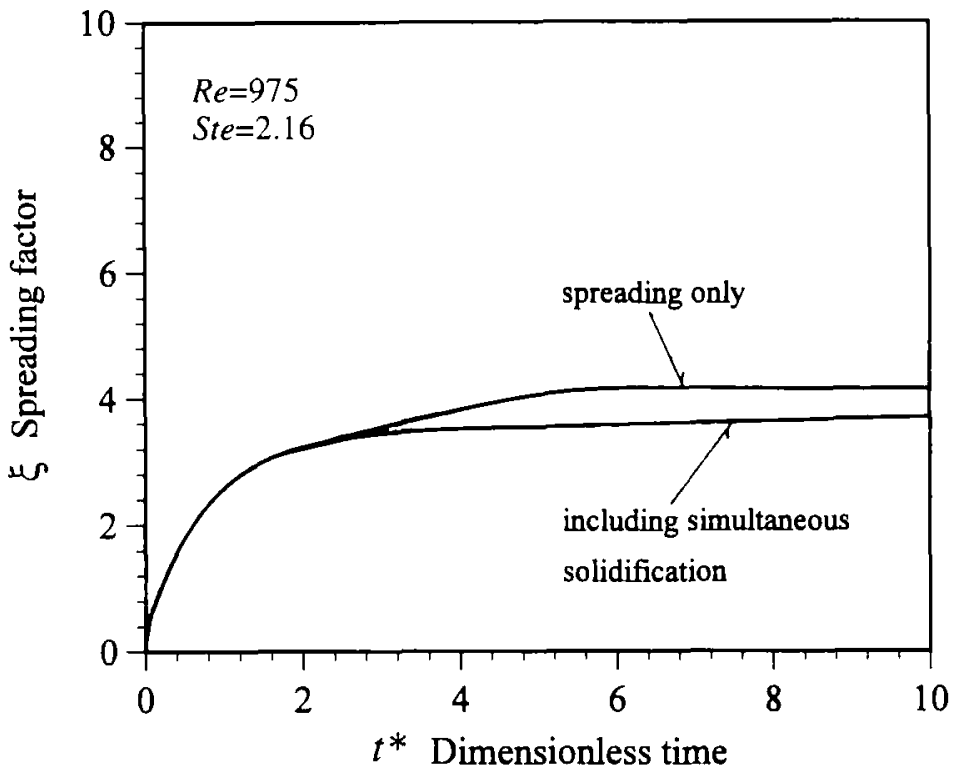

a)

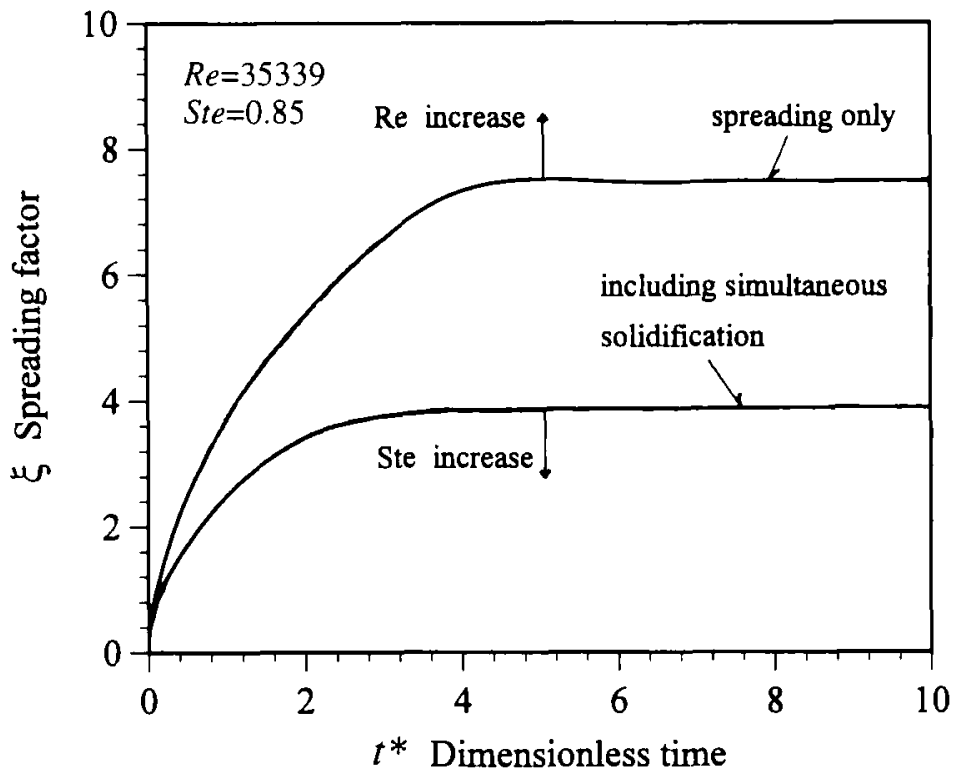

b)

Fig.7. Spreading factor as a function of dimensionless time for : a) an alumina droplet b) a tin droplet ; cases 2 and 3 of Table 1, respectively. (arrows indicate where curves will move if $R e$ and Ste are increased) 


\section{CONCLUSIONS}

A mathematical model for predicting the deformation and simultaneous solidification process of a droplet impinging on a flat substrate was developed in this work. Thermal contact resistance at the substrate was permitted. The flow model is based on the full Navier-Stokes equations, while the solidification model is based on a one-dimensional energy equation. The main features and findings of this work are as follows :

-Close agreement was found between our results and the available experimental data for the spreading of water droplets. This agreement validates the assumptions made in formulating the numerical model.

-The results were compared with the available experimental data for spreading and simultaneous solidification of superheated tin droplets. Close agreement was obtained when a thermal contact resistance of $1.2 \times 10^{-6}$ $m^{2} K W^{I}$ was assumed.

-When the thermal contact resistance was around $1 \times 10^{-6} \mathrm{~m}^{2} \mathrm{KW}^{-1}$, the final splat diameter was extremely sensitive to the magnitude of the contact resistance.

-Simultaneous solidification causes a reduction in the final splat diameter. This effect is more pronounced for high $R e$ and/or high Ste numbers. Previous studies ${ }^{(1,3,4)}$ in which the solidification was considered only after the completion of droplet spreading are only valid for low $R e$ and/or low Ste cases.

-The spreading process in DC plasma spraying is approximately 4 times faster than in RF plasma spraying, a factor which is equal to their impact velocity ratio.

-The time required for spreading and solidification in DC plasma spraying was typically one order of magnitude smaller than in RF plasma spray process.

-Compared to RF plasma spraying, the solidified splats in DC plasma spraying had larger diameters and were of uniform thickness.

\section{ACKNOWLEDGEMENTS}

Financial support of Natural Sciences and Engineering Research Council of Canada is gratefully acknowledged. In addition, the first author wishes to acknowledge financial assistance provided by the Ministry of Culture and Higher Education of Iran.

\section{REFERENCES}

1. T. Bennett and D. Poulikakos, J. of Materials Science 28, 963-970 (1993).

2. M. Pasandideh-Fard, and J. Mostaghimi, Proceedings of the 7 th National Thermal Spray Conference, Boston, Massachusetts, June 1994, pp. 405-414.

3. T. Bennett and D. Poulikakos, J. of Materials Science 29, 2025-2039 (1994).

4. B. Kang, Z. Zhao and D. Poulikakos, J. of Heat Transfer 116, 436-445 (1994). 
5. J. Madejski, Int. J. Heat and Mass Transfer 19, 1009-1013 (1976).

6. S. C. Marchi, H. Liu, A. Sickinger, E. Muhleberg, E. J. Lavernia, and R.H. Rangel, J. of Materials Science 28, 3313-3321, (1993).

7. T. Watanabe, I. Kuribayashi, T. Honda and A. Kanzawa, Chemical Engineering Science 47, 3059-3065 (1992).

8. H. Liu, E.J. Lavernia and R. Rangel, Atomization and Sprays 4, 369-384, (1994).

9. H. Liu, E. J. Lavernia and R. Rangel, International Mechanical Engineering Congress and Exhibition of the Winter Annual Meeting, Chicago, Illinois, November 1994.

10. H. Liu, E. J. Lavernia and R. Rangel, J. Physics. D.: Appl. Physics, 26, 1900-1908, (1993).

11. G. Trapaga, E. F. Matthys, J. J. Valencia and J. Szekely, Metallurgical Transactions 23B, 701-718, (1992).

12. C. Moreau, P. Cielo and M. Lamontagne, J. Thermal Spray Technology 1, 317-323 (1992).

13. C. Moreau, M.Lamontagne and P.Cielo, Surface and Coating Technology 53, 107-114 (1992).

14. C. Moreau, P.Gougeon and M. Lamontagne, J. Thermal Spray Technology 4, 25-33 (1995).

15. S. Fantassi, M. Vardelle, A. Vardelle, A. Denoirjean and P. Fauchais, Proceedings of the 11 th Int. Symposium on Plasma Chemistry, Loughborough, England, Aug. 1993, pp. 186-191.

16. L. Bianchi, M. Mellali, A. Grimaud, P. Fauchais and P. Lucchese, Proceedings of the 11 th Int. Symposium on Plasma Chemistry, Loughborough, England, Aug. 1993, pp. 172-177.

17. L. Bianchi, A. Grimaud, F. Blein, P. Lucchese and P. Fauchais, J. Thermal Spray Technology 4, 59-66 (1995).

18. M. Vardelle, A. Vardelle, A. C. Leger, P. Fauchais, and D. Gobin, J. Thermal Spray Technology 4, 50-58 (1995).

19. C. Moreau, P. Cielo, M. Lamontagne, S. Dallaire, J. C. Krapez and M. Vardelle, Surface and Coating Technology 46, 173-187 (1991).

20. R. McPherson, Thin Solid Films 83, 297 (1981).

21. B. D. Nichols, C. W. Hirt and R. S. Hotchkiss, Los Alamos Scientific Laboratory, LA-8355, UC-32 and UC-34 (1980).

22. H. S. Carslaw, and J. C. Jaeger, Conduction of Heat in Solids, Oxford University Press, London (1959).

23. L. Boruvka and A.W. Neumann, The J. of Chemical Physics 66, 5464-5476 (1977).

24. S. Chandra, Private communications, Department of Mechanical Engineering, University of Toronto (1994).

25. M. Pasandideh-Fard, Y. M. Qiao, S. Chandra and J. Mostaghimi, , FED-Vol. 218 , Experimental and Numerical Flow Visualization, ASME 1995, pp. 53-61

26. H. Fukanuma and A. Ohmori, Proceedings of the 7th National Thermal Spray Conference, Boston, Massachusetts, June 1994, pp. 563-568.

27. M. Vardelle, A. Vardelle, A. C. Leger and P. Fauchais, Proceedings of the 7th National Thermal Spray Conference, Boston, Massachusetts, June 1994, pp. 555-562. 